\title{
Distribución Topográfica y Manejo de Tumores Neuroendocrinos del Tubo Digestivo
}

\section{*Correspondencia:}

dmedica@solca.med.ec Teléfono [593] 04- 3718300 Ext 2124

Conflicto de intereses: LoS autores declaran no tener conflictos de intereses.

Fondos: Ver la página 33

Recibido: 19 Enero 2019

Aceptado: 11 Marzo 2019

Publicado: 30 Abril 2019

Membrete bibliográfico: Panchana G, Macías P, Zambrano D, Lara M, Panchana G. Distribución Topográfica y Manejo de Tumores Neuroendócrinos del Tubo Digestivo. Rev. Oncol. Ecu 2019;29(1):27-35

ISSN:2661-6653

DOI: https://doi.org/10.33821/281

Copyright Panchana, et al Este artículo es distribuido bajo los términos de Creative Commons Attribution License, el cual permite el uso y

redistribución citando la fuente y -1 n...+nn n winimal

\section{Topographic Distribution and Management of Neuroendocrine Tumors of the Digestive Tube}

\section{Guido Panchana Egüez1* (D), Pamela Macías Fernández1(D), Diego A. Zambrano $^{1-2-3}\left[\right.$, Mauricio Lara Perlaza1 ${ }^{1 D}$, Guido Panchana Coello4}

1. Servicio de Cirugía, Solca-Guayaquil.

2. Universidad de Especialidades "Espíritu Santo" (UESS). Samborondón, Ecuador, Departamento de Postgrados.

3. Association Alsace Andes - Post IRCAD Program, Estrasburgo-Francia.

4. Facultad de Medicina, Universidad Católica Santiago de Guayaquil, GuayaquilEcuador.

\section{Resumen}

Introducción: Los tumores neuroendocrinos (TNE) son un grupo de neoplasias que se originan a partir de células enterocromafínicas, especialmente ubicadas en el tubo digestivo. El objetivo del presente estudio es escribir la distribución topográfica, el manejo multidisciplinario y diagnóstico patológico según la OMS de los tumores neuroendocrinos del tubo digestivo.

Métodos: El presente es un estudio descriptivo, retrospectivo de los casos con diagnóstico anatomopatológico confirmado de tumor neuroendocrino localizados en el tubo digestivo entre enero del 2011 a diciembre del 2018 en el Instituto Oncológico Nacional del Ecuador- SOLCA de Guayaquil. Se describe topografía y tipo de tratamiento establecido con frecuencias y porcentajes.

Resultados: Ingresaron al estudio 21 casos. La localización más frecuente fue el apéndice cecal $n=8$ (38\%), en estómago $n=4(19 \%)$, intestino delgado $n=3(14 \%)$. El diagnóstico patológico en estadio $\mathrm{G} 1$ (65\%); G2 (24\%) y G3 (12\%). La primera línea de tratamiento fue la cirugía con intención curativa n=19 $(90.5 \%)$ y tratamiento endoscópico $n=2(9.5 \%)$. Los pacientes sometidos a cirugía, $n=16 / 19 ;(84 \%)$ obtuvieron niveles de resección 0 (R0) y permanecieron en observación clínica, los demás sujetos en el estudio recibieron tratamiento adyuvante con somatostatina sola $n=1(4.8 \%)$ o combinación de somatostatina y radioterapia $\mathrm{n}=2(9.5 \%)$. 
Conclusión: El diagnóstico de tumor neuroendocrino de tubo digestivo es una etiología oncológica poco frecuente. El tratamiento quirúrgico en esta serie de casos está enfocada en una acción curativa de tipo quirúrgico.

Palabras Claves: TUMOR CARCINOIDE, CARNIMONA NEUROENDÓCRINO, TRACTO GASTROINTESTINAL

DOI: $10.33821 / 281$

\section{Abstract}

Introduction: Neuroendocrine tumors (NETs) are a group of neoplasms that originate from enterochromaffin cells, especially located in the digestive tract. The objective of the present study is to write the topographic distribution, the multidisciplinary management and pathological diagnosis according to the $\mathrm{WHO}$ of the neuroendocrine tumors of the digestive tract.

Methods: This is a descriptive, retrospective study of the cases with a confirmed anatomopathological diagnosis of neuroendocrine tumor located in the digestive tract between January 2011 to December 2018 at the National Oncology Institute of Ecuador- SOLCA of Guayaquil. The topography and type of treatment established with frequencies and percentages are described.

Results: 21 cases were entered into the study. The most frequent location was the cecal appendix $\mathrm{n}=$ $8(38 \%)$, stomach $n=4(19 \%)$, small intestine $n=3(14 \%)$. The pathological diagnosis in stage $\mathrm{G} 1(65 \%)$; G2 $(24 \%)$ and G3 (12\%). The first line of treatment was surgery with curative intent $n=19(90.5 \%)$ and endoscopic treatment $n=2(9.5 \%)$. Patients undergoing surgery, $n=16 / 19 ;(84 \%)$ obtained resection levels 0 (R0) and remained under clinical observation, the other subjects in the study received adjuvant treatment with somatostatin alone $\mathrm{n}=1(4.8 \%)$ or combination of somatostatin and radiotherapy $\mathrm{n}=$ $2(9.5 \%)$.

Conclusion: The diagnosis of neuroendocrine tumor of the digestive tract is a rare oncological etiology. The surgical treatment in this series of cases is focused on a surgical action of a surgical type.

Keywords: CARCINOID TUMOR, CARCINOMA, NEUROENDOCRINE, GASTROINTESTINAL TRACT

DOI: $10.33821 / 281$

\section{Introducción}

Los tumores neuroendocrinos (TNE) son un grupo de neoplasias que se originan a partir de células enterocromafínicas, las mismas que se encuentran distribuidas en todo el cuerpo, especialmente en submucosa del intestino y del pulmón. Fueron identificados por primera vez como un tipo específico de crecimiento distinto a mediados de 1800 . Langhans fue el primero en describir un tumor carcinoide en 1867, y en 1888 fue Lubarsch quien lo registró por primera vez como un tumor nuevo, así dos años después Ransom dio la primera explicación del cuadro clásico de síntomas del síndrome carcinoide [1].

Actualmente bajo el término de TNE se recoge el amplio espectro de estas neoplasias, desde la presentación clásica del tumor carcinoide en un extremo hasta la variedad anaplásica en el otro. Según las nuevas guías se debe utilizar el término TNE como sinónimo de tumor carcinoide (TC). Los cuales incluyen desde neoplasias bien diferenciadas con 
crecimiento lento y potencial bajo de metástasis (TC), hasta neoplasias poco diferenciadas con crecimiento rápido y potencial alto de metástasis (TNE).

La incidencia se ha incrementado de manera importante, según la literatura internacional, al parecer en base a los nuevos estudios diagnósticos realizados [2-3]. La supervivencia general de estos tumores puede variar significativamente del 40 al $60 \%$ a los 50 años dependiendo de su distribución y diferentes factores pronósticos, siendo uno de los más importantes: ubicación del tumor, estadio de la enfermedad y diferenciación histológica, e índice mitótico; las que comprometen el páncreas seguidas de las que afectan el colon, son los de peor pronóstico. Se estima que por lo general los tumores carcinoides se originan de transformaciones malignas de células neuroendocrinas pluripotenciales, siendo este mecanismo en gran parte desconocido; se postula que el daño temprano de las células neuroendocrinas pluripotenciales origina tumores mal diferenciados o de alto grado; mientras que los $\mathrm{G} 1$ y G2, denominados bien diferenciados, se originan a partir de etapas más posteriores $[4,5]$.

En Ecuador no hay estudios que establezcan la incidencia del tumor neuroendocrino en el tubo digestivo y son pocos los centros especializados que permiten establecer el manejo de este en un ambiente multidisciplinario por lo que el objetivo del presente estudio es describir la distribución topográfica, el manejo multidisciplinario y diagnóstico patológico según la OMS de los tumores neuroendocrinos del tubo digestivo.

\section{Materiales y Métodos}

Este es un estudio retrospectivo de todos los casos de tumores neuroendocrinos del tubo digestivo confirmados por diagnóstico histopatológico de resecciones quirúrgicas y endoscópicas; recolectados desde enero del 2011 a diciembre del 2018 en el Instituto Oncológico Nacional del Ecuador- Solca de Guayaquil. La fuente de datos fue el Registro de Tumores de Solca-Guayaquil (2011-2018).

El universo estuvo conformado de todos los pacientes que ingresaron al servicio con tumores neuroendócrinos que tuvieron todos los datos analizables. El muestreo fue no probabilístico de tipo intencional.

Las variables documentadas fueron sexo, edad, tipo de cáncer clasificado, localización del tumor en el tubo digestivo, tratamiento quirúrgico, tratamiento endoscópico, diagnóstico histopatológico según la OMS (Ki67 y conteo mitótico) y tratamiento adyuvante. Por ser estudio retrospectivo y descriptivo el estudio no requirió consentimiento informado y aprobación del comité de ética de la institución.

Se utiliza análisis descriptivo con frecuencias y porcentajes.

\section{Resultados}


Se identificaron 21 casos de TNE del tubo digestivo, 6 hombres (29 \%) y 15 mujeres (71\%) con un promedio de edad de 47 años, con un rango desde los 19 años hasta los 70 años.

La sintomatología clínica inicial correspondió a síntomas generales $\mathrm{n}=9$ (43\%) como dolor abdominal, melena, pérdida de peso; seguida de síntomas específicos $n=7$ (33\%) como diarrea, rubor y disnea; y otros síntomas $n=5(24 \%)$.

La localización en orden descendente fue: apéndice cecal $n=8$, (38 \%), estómago $n=4$ (19 $\%)$, intestino delgado $n=3(14 \%)$ de los cuales todos pertenecieron al duodeno, colon y recto n=4 (19\%), por último, el páncreas n=2 (10\%). Figura 1.

Las intervenciones quirúrgicas con intención curativa en los pacientes con tumor neuroendocrino no metastásicos se realizaron según su topografía, así podemos detallar que en estómago se practicaron, gastrectomía subtotal $n=3(14 \%)$ y gastrectomía total $n=1$ (5\%); en páncreas, pancreatectomía distal $n=1(5 \%)$ y enucleación $n=1(5 \%)$; en intestino delgado, resección con exéresis tumoral $n=1$ (5\%); I y duodenopancreatecnomía cefálica n=2 (9\%); en apéndice cecal, apendicectomía terapéutica $n=6(29 \%)$ y apendicectomía profiláctica $n=2(9 \%)$; en colon y recto, resección anterior baja $n=3(14 \%)$ y resección transanal $n=1$ (5\%). Figura 1.

Figura 1. Distribución topográfica y tratamiento quirúrgico en TNE del tubo digestivo.

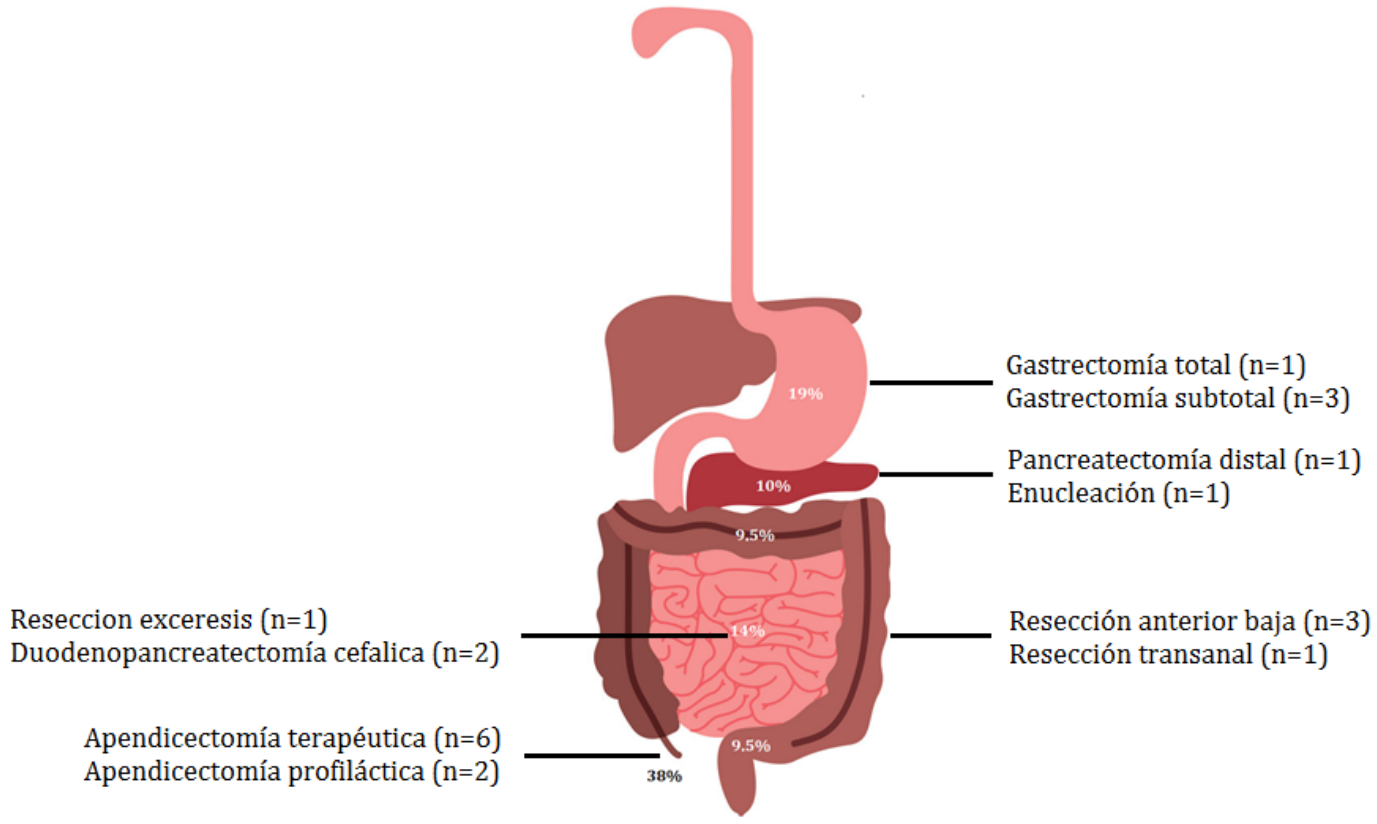

Image License for Reproduction from VectorStock.com/15381431

El nivel de resección quirúrgica y el tratamiento postoperatorio indicado por el equipo multidisciplinario para los pacientes del estudio comprendió aquellos con nivel de resección R0 n=16 (84\%), cuyo manejo postoperatorio fue la observación clínica; mientras que en los pacientes con nivel de resección quirúrgica $\mathrm{R} 1 \mathrm{n}=3$ (16\%), 1 caso recibió quimioterapia sola, 
y los otros recibieron quimioterapia con análogos de la somatostatina y radioterapia complementaria.

Los resultados de patología en base al estadio según la Organización Mundial de la Salud (OMS, 2010) se estableció el estadio G1 n=14 (67\%); estadio G2 n=5 (24\%) y el estadio G3 $\mathrm{n}=2$ (9\%) en el total de casos de TNE del tubo digestivo (Figura 2).

Figura 2. Clasificación de Neoplasias Neuroendocrinas Digestivas de la Organización Mundial de la Salud (OMS) 2010.

\begin{tabular}{|c|c|c|}
\hline & $\begin{array}{c}\text { Índice Mitótico } \\
\text { (por 10 CGA) }\end{array}$ & Ki67 (\%) \\
\hline G1 & $<2$ & $=2$ \\
\hline G2 & $2-20$ & $3-20$ \\
\hline G3 & $>20$ & $>20$ \\
\hline
\end{tabular}

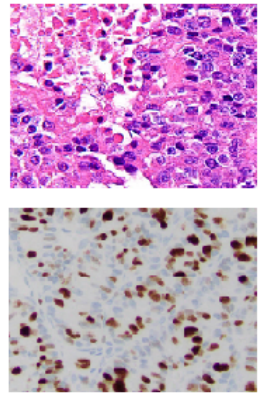

$1 \mathrm{CGA}=2 \mathrm{~mm}^{2}$

Se cuentan 40 campos en las zonas de densidad mitótica más altas.

Porcentaje de células positivas por 500-2000 células en la zona de mayor marcaje nuclear.

Fuente: WHO Classification of Tumours of the Digestive System. Fourth Edition (2010)

\section{Discusión}

La incidencia del tumor neuroendocrino representa en los estudios internacionales alrededor del $2 \%$ de los tumores que afectan el tubo digestivo [4-5]. Este estudio demuestra nuestra experiencia entre el 2011 al 2018 en el Instituto Oncológico Nacional del EcuadorSOLCA de Guayaquil.

Hemos comparado nuestro estudio con aquellos internacionales como el Surveillance Epidemiology End Results (SEER) [6], en el que se evidencia el intestino delgado (38 \%) como la topografía de tumor digestivo neuroendocrino de mayor incidencia, contrastando con nuestro estudio donde los tumores neuroendocrinos de apéndice cecal ocuparon el primer lugar $(n=8,38 \%)$ y el de páncreas $(n=2,10 \%)$.

La edad osciló entre los 19 años hasta los 70 años, con una media de 47 años; con una diferencia de 10 años en comparación a otros estudios. (7-8) Las mujeres fueron las más afectadas $(n=15,71 \%)$ a diferencia con los estudios ERG, TNCS y SEER en que la relación sexo masculino / femenino fue del $2 / 0.92$ respectivamente $[6,9,10]$.

El tratamiento quirúrgico al igual que estudios comparativos se indicó en base a su topografía, tamaño del tumor, presentación al momento del diagnóstico sea este localizado, regional o metastásico. Con respecto al manejo de los 4 casos de tumor neuroendocrino de estómago; se procedió a realizar gastrectomía subtotal en el $75 \%$ de los casos a aquellos con lesiones tumorales menor de $1 \mathrm{~cm}$, mientras que en la restante gastrectomía total fue 
sometida a aquella lesión mayor de $1 \mathrm{~cm}$, mientras que los estudios determinan que las lesiones menores de $1 \mathrm{~cm}$ y hasta $1.5 \mathrm{~cm}$ se sugiere tratamiento endoscópico tipo mucosectomía. A pesar de la controversia muchos grupos aún realizan tratamiento quirúrgico a partir de $1 \mathrm{~cm}$ del tamaño tumoral, o en menores de $1 \mathrm{~cm}$ en caso de lesiones múltiples $[11,12]$.

El tratamiento optado en los casos de TNE de apéndice cecal contrasta con respecto a las normas ya que se optó por tratamientos más conservadores en el $50 \%$ de los pacientes, ya que en base al grado de infiltración y el tamaño tumoral debieron haberse complementado su tratamiento quirúrgico diferido con hemicolectomía derecha, aunque cabe recalcar que la supervivencia hasta la culminación del estudio fue del $100 \%$ sin signos de recidiva tumoral [13-14].

Se demostró que el manejo del tumor neuroendocrino tiene como componente principal el tratamiento quirúrgico, al observar nuestra experiencia con respecto al tratamiento postoperatorio optado, ya que sólo aquellos pacientes con nivel de resección quirúrgica R1 fueron beneficiarios de tratamiento complementario con quimioterapia y radioterapia [1516].

\section{Conclusiones}

En el manejo del TNE del tubo digestivo debe realizarse con un equipo multidisciplinario, considerar que el tratamiento quirúrgico es el que tiene el mayor potencial curativo, debiendo siempre considerar la localización anatómica y factores propios del tumor; y definir la necesidad de tratamiento adyuvante.

\section{Agradecimientos}

Reconocemos a las personas que participaron indirectamente en el estudio tales como el personal técnico, pacientes y personal del Instituto Oncológico Nacional "Dr. Juan Tanca Marengo", Solca-Guayaquil.

\section{Información adicional}

\section{Abreviaturas}

SEER: Surveillance Epidemiology End Results.

TC: tumor carcinoide.

TNE: Tumores Neuroendócrinos.

\section{Nota del Editor}

La Revista Oncología Ecu permanece neutral con respecto a los reclamos jurisdiccionales en mapas publicados y afiliaciones institucionales.
Archivos Adicionales 
Ninguno declarado por los autores.

Fondos

Los fondos de la investigación fueron propios de los autores del presente artículo.

\section{Disponibilidad de datos y materiales}

Existe la disponibilidad de datos bajo solicitud al autor de correspondencia. No se reportan otros materiales.

\section{Contribuciones de los autores}

GPE, PMF, DAZ realizaron la idea de investigación, revisión bibliográfica. MLP, GPC, recolección de datos, escritura del artículo. GPE realizó el análisis crítico del artículo. DAZ realizó las correcciones editoriales. Todos los autores leyeron y aprobaron la versión final del artículo.

\section{Aprobación de ética y consentimiento para participar}

No aplica a este estudio.

\section{Consentimiento para publicación}

No aplica.

\section{Información de los autores}

Guido Panchana Egüez, Médico del Departamento de Cirugía del Instituto Oncológico Nacional del Ecuador “Dr. Juan Tanca Marengo” -SOLCA, Guayaquil-Ecuador.

밈odica@solca.med.ec (593)-4-371-8300 ext. 2124

(iD) https://orcid.org/0000-0002-8628-0538

Diego A. Zambrano, Médico del departamento de Cirugía, Instituto Oncológico Nacional del Ecuador "Dr. Juan Tanca Marengo" -SOLCA, Guayaquil-Ecuador. Association Alsace Andes - Post IRCAD Program, Estrasburgo-Francia. Universidad de Especialidades Espíritu Santo (UEES), Guayaquil-Ecuador.

○@ dizampe@gmail.com (593)-4-371-8300 ext. 2123 
Pamela Macías Fernández, médico del departamento de Cirugía del Instituto Oncológico Nacional del Ecuador “Dr. Juan Tanca Marengo” -SOLCA, Guayaquil-Ecuador.

¿@panchanag@gmail.com (593)-4-371-8300 ext. 2180

Mauricio Lara Perlaza, médico del departamento de Cirugía, Instituto Oncológico Nacional del Ecuador "Dr. Juan Tanca Marengo" -SOLCA, Guayaquil-Ecuador.

Guido Panchana Coello, profesor de la Facultad de Medicina, Universidad Católica Santiago de Guayaquil, Guayaquil-Ecuador.

panchanag@gmail.com $\mathbf{C}_{(593)-99-511-6848}$

iDttps://orcid.org/0000-0003-0543-2243

Revisiones por pares

Acceda a la revisión de pares académicos en el siguiente enlace: https://publons.com/review/4516348/

\section{Referencias}

Abreviaturas en la referencias DOI: Digital Object Identifier PMID: PubMed Identifier SU: Short URL
1. Modlin IM, Lye KD, Kidd M. A 5-decade analysis of 13,715 carcinoid tumors. Cancer. 2003;97(4):934-59. DOI: $10.1002 /$ cncr.11105

2. Oberhelman Ha Jr, Nelsen Ts. Surgical Consideration In The Management Of Ulcerogenic Tumors Of The Pancreas And Duodenum. Am J Surg. 1964;108:132-41.

3. Uhr JH, Shah J, Warner RR, Divino CM. Racial Disparities in Clinical Presentation and Surgical Outcomes in Patients with Small Bowel Carcinoid Tumors. Am Surg. 2016;82(4):E89-92. PMID: 27097615.

4. Yao JC, Hassan M, Phan A, Dagohoy C, Leary C, Mares JE, et al. One hundred years after "carcinoid": epidemiology of and prognostic factors for neuroendocrine tumors in 35,825 cases in the United States. J Clin Oncol. 2008;26(18):3063-72. DOI: 10.1200/JC0.2007.15.4377.

5. Kim JY, Hong SM. Recent Updates on Neuroendocrine Tumors From the Gastrointestinal and Pancreatobiliary Tracts. Arch Pathol Lab Med. 2016;140(5):437-48. DOI: 10.5858/arpa.2015-0314-RA

6. Duggan MA, Anderson WF, Altekruse S, Penberthy L, Sherman ME. The Surveillance, Epidemiology, and End Results (SEER) Program and Pathology: Toward Strengthening the Critical Relationship. Am J Surg Pathol. 2016;40(12):e94-e102. DOI: 10.1097/PAS.0000000000000749

7. Mocellin S, Nitti D. Gastrointestinal carcinoid: epidemiological and survival evidence from a large population-based study ( $\mathrm{n}=25$ 531). Ann Oncol. 2013;24(12):3040-4 DOI: 10.1093/annonc/mdt377

8. Anthony LB, Kulke MH, Caplin ME, Bergsland E, Öberg K, Pavel M, et al. Long-Term Safety Experience with Telotristat Ethyl Across Five Clinical Studies in Patients with Carcinoid Syndrome. Oncologist. 2019;pii:theoncologist.2018-0236. DOI: 10.1634/theoncologist.2018-0236

9. Teunissen JJ, Kwekkeboom DJ, de Jong M, Esser JP, Valkema R, Krenning EP. Endocrine tumours of the gastrointestinal tract. Peptide receptor radionuclide therapy. Best Pract Res Clin Gastroenterol. 2005 Aug;19(4):595-616. DOI: 10.1016/j.bpg.2005.04.001

10. Godwin JD. Carcinoid tumors. An analysis of 2,837 cases. Cancer. 1975; 36(2):560-9. PMID: 1157019 
11. Ramage JK, Ahmed A, Ardill J, Bax N, Breen DJ, Caplin M, et al. Guidelines for the management of gastroenteropancreatic neuroendocrine (including carcinoid) tumours. Guidelines for the management of gastroenteropancreatic neuroendocrine (including carcinoid) tumours (NETs). Gut. 2012;61(1):6-32. DOI: 10.1136/gutjnl-2011-300831

12. Yoshida M, Tsukamoto Y, Niwa Y, Goto H, Hase S, Hayakawa T, Okamura S. Endoscopic assessment of invasion of colorectal tumors with a new high-frequency ultrasound probe. Gastrointest Endosc. 1995;41(6):587-92. PMID:7672554.

13. Rindi G, Petrone G, Inzani F. The 2010 WHO classification of digestive neuroendocrine neoplasms: a critical appraisal four years after its introduction. Endocr Pathol. 2014;25(2):186-92. DOI: 10.1007/s12022-014-9313-z

14. Shapiro R, Eldar S, Sadot E, Papa MZ, Zippel DB. Appendiceal carcinoid at a large tertiary center: pathologic findings and long-term follow-up evaluation. Am J Surg. 2011;201(6):805-8. DOI: 10.1016/j.amjsurg.2010.04.016

15. Liu EH, Solorzano CC, Katznelson L, Vinik Al, Wong R, Randolph G. AACE/ACE disease state clinical review: diagnosis and management of midgut carcinoids. Endocr Pract. 2015;21(5):534-545. DOI: 10.4158/EP14464.DSC

16. Varas-Lorenzo MJ, Cugat E, Capdevila J, Sánchez-Vizcaíno E. Detección de tumores neuroendocrinos pancreáticos: 23 años de experiencia. Revista de Gastroenterología de México 2019;84(1):18-25. DOI: 10.1016/j.rgmx.2018.02.015 